\title{
Multi factorial challenges in managing a patient with neonatal diabetes
}

\author{
AR Akula, A Karthikeyan, H Stirling \\ Department of Paediatrics, University Hospital Coventry \& Warwickshire NHS Trust, \\ Coventry, United Kingdom
}

Disclosure statement: The authors have nothing to disclose

\section{Case}

We report the case of a term baby born to a diet controlled gestational diabetic mother with a birth weight of $2.8 \mathrm{~kg}$. Baby was persistently hyperglycaemic from day 1 of life, leading to a diagnosis of neonatal diabetes being considered. She was breast-fed on demand and although insulin pump therapy was discussed, it was felt not to be suitable, as language barriers would have meant that parents would have had difficulty recognising pump failure/alarms. Twice daily subcutaneous injections of Isophane Insulin (Insulatard) were commenced, although there have been no studies on efficacy of Insulatard in neonates. The small doses of insulin required were administered by diluting the insulin with a suitable diluting medium. At 8 months of age our patient is thriving and developing well with no hospital admissions. She is on 0.2 units $/ \mathrm{kg} /$ day of insulin with most recent glycosylated haemoglobin ( $\mathrm{HbA1C}$ ) being $48 \mathrm{mmol} / \mathrm{mol}$. No known genetic mutation has been identified.

\section{Challenges}

Providing training for the family on self-management called for significant input from the diabetes specialist nurses. Telephone follow up was challenging due to language barriers, resulting in the need for frequent home visits and hospital appointments for close monitoring.

\section{Conclusion}

The management of neonatal diabetes is challenging for many reasons. Blood glucose monitoring can be technically demanding for carers and traumatic for infants. Insulin requirements and insulin sensitivity can be very variable. As has been highlighted by others, the small volumes of insulin required cannot be delivered using standard insulin formulations and these do not provide insulin delivery profiles that complement the feeding patterns and glycaemic excursions of newborns (1). Conventional insulin delivery devices are also often not useful. Our case highlights that physiological and pharmocological factors as well family and social factors influence treatment planning.

\section{References}

1.Beardsall K, Pesterfield CL, Acerini CL. Neonatal diabetes and insulin pump therapy, Arch Dis Child Fetal Neonatal Ed. 2011 May;96(3):F223-4.

2.Passanisi S, Timpanaro T, Lo Presti D, Mammi C, Caruso-Nicoletti M; Treatment of transient neonatal diabetes mellitus: insulin pump or insulin glargine? Our experience, Diabetes Technol Ther.

2014;16(12):880-4.

3.Bharucha T, et al. Neonatal diabetes mellitus: Insulin pump as an alternative management strategy. J Paediatr Child Health2005;41(9-10):522-6 